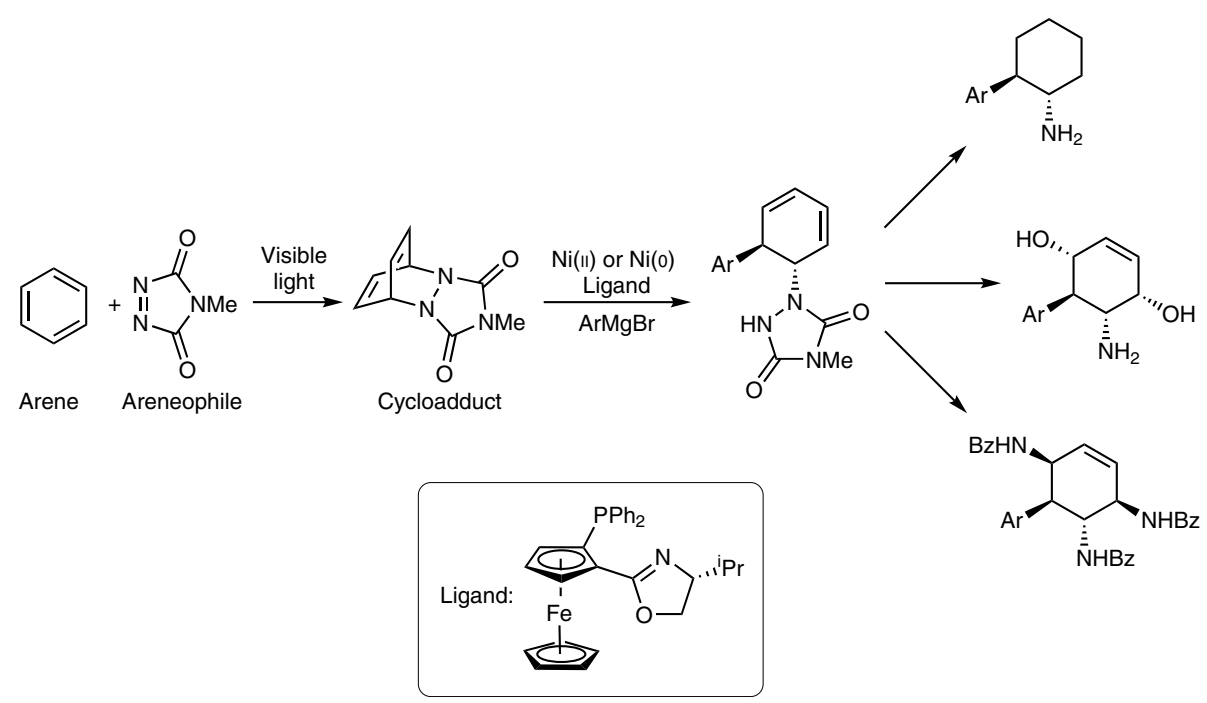

Functionalizing benzene and related aromatics is a challenge - additions to the ring system involve breaking the aromaticity. David Sarlah and co-workers at the University of Illinois have been examining this problem for some time, and have previously reported an areneophile capable of dearomative cycloadditions to a range of arenes. The resulting cycloadduct has proven to be a versatile intermediate that can further be derivatized to give functionalized 1,3- and 1,4-cyclohexadienes.

Some earlier work by the group had shown that nickel-catalysed additions of a Grignard reagent to this intermediate could give a trans-1,2-carboamination product. Now they examine this process in detail, demonstrating that it can be a general route to enantioenriched amines (pictured).

Air-stable and inexpensive Ni(II) salts could be used in place of the less user friendly $\mathrm{Ni}(0)$ species previously employed. With respect to the Grignard component, a range of aryl, vinyl and naphtyl groups could be used to give the 1,2-addition products as single diastereomers with good yields and high enantiomeric excesses. Scale-ups to multigram scale were shown to be possible without any loss in enantioselectivity.

For the arene component, substituents on the ring systems proved more challenging. Unlike benzene and naphthalene, once extra substituents were added to the starting materials the enantioselective desymmetrization step was no longer effective. However, the racemic products could still be formed as single diastereomers using $\mathrm{Ni}(\mathrm{cod})_{2}$ and an achiral bisphosphine (dppf).

Finally, the functionalized 1,3-cyclohexadiene products were further derivatized. The urazole could be converted into a primary amine (or removed completely), and the diene could be reduced or undergo further additions to give products with up to four contiguous stereocentres. Overall it looks like a powerful method to quickly introduce complexity into compounds that typically display a limited range of reactivity.

\section{Enda Bergin}

Published online: 16 April 2018 https://doi.org/10.1038/s41929-018-0064-y 